\title{
Effect of lifestyle Promotion Booklet on the Outcomes of Patients with Insulin Dependent Diabetes Mellitus (IDDM)
}

\author{
Amira Sameh Ahmed ${ }^{1}$, Zeinab Abd El-lateef Mahamad², Muhammad Hossam Eldeen Hassan ${ }^{3}$, Sahra Zaki \\ Azer $^{4}$ \\ 1. B.Sc.Ng, Faculty of Nursing, Assiut University, Egypt. \\ 2. Professor of Medical Surgical Nursing, Faculty of Nursing, Assiut University Egypt. \\ 3. Professor of Internal Medicine, Faculty of Medicine, Assiut University Egypt. \\ 4. Assistant Professor of Medical Surgical Nursing, Faculty of Nursing, Assiut University Egypt.
}

\begin{abstract}
Background: Type 1 diabetes is an autoimmune disease with a strong genetic component. It can occur at any age Aim: To evaluate the effect of lifestyle promotion on outcomes of patients with insulin dependent diabetes mellitus. Research design: Pre/posttest research design was utilized. Setting: The study was conducted in the internal medical department \& outpatient clinic at Assuit University Hospital. Samples included 50 adult patients selected according to the following criteria: Conscious patient, age range between 18-65 years, from both sexes and insulin dependent diabetes mellitus. Tools: Patient interview questionnaire. It includes: demographic data and health promotion lifestyle. Based on the analysis of the assessment tools health promotion lifestyle booklet was developed. Results: More than have of the studied sample were female and there mean age were 49.220 \pm 14.226 . Patients' level of knowledge was increased after the implementation of the booklet; particularly in the areas of the health responsibility, physical activity, interpersonal relations, and nutrition. Conclusion: There are statistical significance differences for studied samples as regard to life style subscale. Recommendation: It is important to educate patients about how to modify life style to healthy life, prevent and manage diabetic complications.
\end{abstract}

\section{Keywords: Insulin Dependant Diabetes Mellitus, Life Style Promotion \& Out Comes.}

\section{Introduction}

Diabetes mellitus (DM) is a syndrome caused by an imbalance between insulin supply and demand. It is characterized by hyperglycemia and associated with abnormal carbohydrate, fat and protein metabolism (Ozougwu et al., 2013).

The prevalence of DM has increased dramatically in most countries in the world. Affecting more than 415 million adults in the world (Ogurtsova et al., 2017). Recently, it is estimated that the number of DM patients will increase from 171 million to 366 million between the years 2000 and 2030 (Ogurtsova et al., 2017).

Blood-glucose level was controlled by the both insulin and glucagon, pancreatic endocrine hormones within the body in an adequate level based on the body needs. Normally, insulin is secreted by the Bcells found at the islets of Langerhans in response to high levels of blood sugar. It provide the capability of muscle, red blood cells, and fat cells to absorb sugar out of the blood and consume it in other metabolic processes, which restore the sugar levels to the normal level ( Röder et al., 2016).

Diabetes can be classified into the following general categories: Type 1 diabetes due to autoimmune $\beta$-cell destruction, usually leading to absolute insulin deficiency, Type 2 diabetes due to a progressive loss of $\beta$-cell insulin secretion frequently on the background of insulin resistance and gestational diabetes mellitus (GDM)(ADA, 2016).

The management of diabetes requires patients to perform complex self-care regimens, including weight reduction, frequent blood glucose monitoring, taking oral and/or insulin medications, engaging in physical activity, adhering to diabetes nutrition guidelines, and attending clinic appointment for follow up (Garber et al., 2013).

Health promotion lifestyles refer to the behaviors of individuals, families, communities, and societies forwards the promotion of peace, happiness, and the realization of health potential as, any activity that one could may take to achieve a higher level of health, self-realization, peace and happiness (Pender, 2011). Patients and care providers should focus together on how to optimize lifestyle from the time of the initial comprehensive medical evaluation, throughout all subsequent evaluations and follow-up, and also during the assessment of complications and management of comorbid conditions in order to enhance care of patients with DM (Kalyani et al., 2018).

\section{Significance of the study}

According to the medical records in the year of (2018) , at Assiut university hospitals the total number of diabetic patients admitted to the internal medicine department were 295 cases. From the 
researcher's clinical experience it was observed that the patients who were diagnosed with IDDM are in need for health promotion lifestyle pattern in a form of booklet to improve their outcomes. This study was conducted to provide patients with health promotion lifestyle booklet that provide information on how to stay as healthy as possible.

\section{Aim of the study}

To evaluate the effect of lifestyle promotion on outcomes of patients with insulin dependent diabetes mellitus (IDDM).

Study hypotheses

To fulfill the aim of this study, the following research hypotheses are formulated:-

Patient's information outcomes about insulin dependent diabetes mellitus, health responsibility, nutrition, medications, activity and exercises will be better following the implementation of the health promotion lifestyle booklet.

\section{Subjects \& Method \\ Research design}

Pre/posttest research design was utilized to conduct this study.

\section{Setting:}

The study was conducted in the internal medical department \& outpatient clinic at Assiut University Hospital.

\section{The Subject (Patients)}

A purposive sample included 50 adult patients. Who were selected according to the following criteria: Conscious patient, age range between (18-65) years, from both sexes, and diagnosed as insulin dependent diabetes mellitus.

\section{Sample size}

According to assessment health promotion life style (HPL) mean (pre) 78.70, compared with mean in (Post) 88.56 standard deviation 15.30 in pre and 10.70 post according to the significance level of 0.05 and a power of 0.80 , it was calculated that the sample size of 29 cases at minimum number.

\section{Tools of data collection}

After review of literature and consultation of supervisors, tools were prepared and filled by the researcher using the interview methods. Two tools of the study utilized for data collection and were include the following

\section{Tool 1:Patient questionnaire sheet}

This sheet was developed by the researcher based on current national and international literature, it include two parts:

\section{Part I: Socio demographic data for patient} including age, sex, occupation, marital status, diagnosis and health history.
Part II: Health promotion lifestyle (HPL) Pre / Post

The original HPLP-II instrument (in English) was developed and revised by (Walker et al., 1987), to assess the lifestyle pattern of patients with IDDM. The Health promotion lifestyle instrument has 35 health-promoting behaviors categorized into four health-promoting lifestyle subscales: (1) health responsibility (HR), (2) physical activity (PA), (3) interpersonal relations (IR) and (4) nutrition (N). Four point response scale consisting of $1=$ never , 2 $=$ sometimes , $3=$ often , and $4=$ routinely were used to determine the frequency of each behavior. A mean of $>2.50$ was considered to be a positive response (Wei et al., 2012). Operational definition:

- Health promotion lifestyle: define as multidimensional pattern of self-initiated actions and perceptions that serve to maintain or enhance the level of wellness, self-actualisation and fulfillment of the individual (Alpar et al., 2008).

- lifestyle promotion outcomes: it include information about diabetes, health responsibility, nutrition, activity and exercises, medications.

- Insulin dependent diabetes mellitus (IDDM): is an autoimmune disease with a strong genetic component. Type1 diabetes mellitus is characterized by destruction of pancreatic $\beta$-cells, culminating in absolute insulin deficiency (Noble \& Valdes, 2011).

After data collection and analysis and based on the patients needs assessment the booklet was developed. Health promotion lifestyle booklet:

It was developed by the researcher after extensive relevant literature review and according to the patients' needs and was containing the following:

- Information about diabetes mellitus, health responsibility, nutrition, medications, activity and exercises.

\section{Methods}

This study was carried out in three phase: Involve the following

\section{I.Preparatory phase ( assessment and planning phase )}

An official approval letter was obtained from the dean of the faculty of nursing, Assiut University to the head of medical department at Assiut University Hospital.

The researcher reviewed related literature of the current study, local \& international, using text books, articles, and scientific magazines. The proposed study setting was assessed for the numbers of patients in the internal medical department. The designed health promotion lifestyle booklet was developed by the researcher, after extensive literature review 
considering patients' needs and their levels of understanding.

\section{Content validity and Reliability}

The validity was done for the study tool by five experts in medical and nursing staff for clarity, relevance, comprehensiveness, applicability and easiness.

The final form of the tools was designed and tested for reliability by using internal consistency for the tools measured using Cronbach test, the tools proved to be reliable at $(0.73)$.

\section{A pilot study}

A pilot study carried out in june 2018 to test the feasibility and applicability of the study tools and conducted on $10 \%$ of the sample (5 patients) of sample. It had also provided an estimate of time needed to fill out the tools. The data obtained from the pilot study were analyzed and change was done in the study tool. Patients participating in the pilot study were included in the main study.

\section{Implementation phase ( work field)}

Each patient was interviewed individually after receiving medical management. Oral consent was obtained. The study was carried out at morning and afternoon shifts for all available patients.The researcher introduce herself to initiate line of communication, explain the nature and purpose of the designed health promotion lifestyle booklet to the selected patients who are willing to participate in the study. Fill out the questionnaire sheet tool (1) to assess the patients knowledge before application of designed teaching booklet and scheduled with them the teaching session.

The researcher interview patients 5 days per week and one patient every day. The each patient toke 10 minutes for collection of data pre implementing booklet session. And 10 minutes for collection of evaluation data.

The period of data collection 6 months from July 2018 to January 2019.

\section{Booklet session implementation}

All patients received the contents of the designed health promotion lifestyle booklet by the researcher herself. Designed health promotion lifestyle booklet was conducted through 4 sessions for each patient in addition to the pre assessment session and the duration of each session was range between 20 to 30 minutes. Each session usually started by a summary of what had been taught during the previous session and the objectives of the new session. After each session there was 5 minutes for discussion and feedback. Reinforcement of teaching was performed according to patient's needs to ensure their understanding. Each patient in the study group obtains a copy of the teaching booklet in Arabic language. The researcher used pictures for illustration and diagram to facilitate the information to the patient.

First session: Information about the definition of diabetes mellitus, its types, signs and symptoms, and risk factors.

Second session: Included information about health responsibility as regular follow up, measuring blood pressure, doing laboratory investigations, nutrition ( types of foods \& fluids and number of meals).

Third session: It inform the patient about activities of daily living, practicing exercise, duration of the exercise, information about the importance of exercise, types and how to perform these exercise (aerobic $\&$ anaerobic). At the end of this session the teaching booklet was given to the patients to be used as a guide for them.

Four session: It included information about medications such as type, dose, time, route, precautions \& side effects. Instruct the patients about how to be self management in to his hypo or hyperglycemia

\section{III.Evaluation phase}

The patient was evaluated after three months from date of discharge from hospital to evaluate the effectiveness of the teaching protocol on using part 2 from the study tool to compeer between the pre and post assessment.

\section{Ethical considerations:}

The study will follow the common ethical guidelines of clinical research according to the principles of Helinki declaration for medical researche (Helsinki, 1996).

\section{The statistical design}

The data obtained had reviewed, prepared for computer entry, coded, analyzed and tabulated, descriptive statistics include (frequencies and percentages, mean and standard deviation) use Pearson chi-square (cross tabulation), Independent t-test, and one way anova between pre, and post-test after three months $\mathrm{p} \leq 0.01$, were done using computer program (SPSS). version 23. 


\section{Results}

Table (1): Frequency distribution of characteristics of patients $(n=50)$.

\begin{tabular}{|c|c|c|}
\hline Variables & No. & $\%$ \\
\hline $\begin{array}{l}\text { 1. Age: } \\
-\quad 18 \text { less than 30yrs } \\
-\quad 30 \text { less than 50yrs } \\
-\quad 50-65 y r s\end{array}$ & $\begin{array}{c}6 \\
15 \\
29\end{array}$ & $\begin{array}{l}12.0 \\
30.0 \\
58.0\end{array}$ \\
\hline Mean \pm SD & \multicolumn{2}{|c|}{$49.220 \pm 14.226$} \\
\hline $\begin{array}{l}\text { 2. Sex: } \\
-\quad \text { Male } \\
-\quad \text { Female }\end{array}$ & $\begin{array}{l}24 \\
26\end{array}$ & $\begin{array}{l}48.0 \\
52.0 \\
\end{array}$ \\
\hline $\begin{array}{l}\text { 3. Marital status: } \\
-\quad \text { Single } \\
-\quad \text { Married } \\
-\quad \text { Widow }\end{array}$ & $\begin{array}{c}6 \\
40 \\
4\end{array}$ & $\begin{array}{c}12.0 \\
80.0 \\
8.0\end{array}$ \\
\hline $\begin{array}{l}\text { 4. Level of education: } \\
-\quad \text { Not educated } \\
-\quad \text { Read and write } \\
-\quad \text { Primary education } \\
-\quad \text { Preparatory school } \\
-\quad \text { Secondary school } \\
-\quad \text { University } \\
\end{array}$ & $\begin{array}{c}19 \\
11 \\
3 \\
1 \\
11 \\
5\end{array}$ & $\begin{array}{c}38.0 \\
22.0 \\
6.0 \\
2.0 \\
22.0 \\
10.0\end{array}$ \\
\hline $\begin{array}{l}\text { 5. Occupation: } \\
-\quad \text { Not-working } \\
-\quad \text { Farmer } \\
-\quad \text { Student } \\
-\quad \text { Professional } \\
-\quad \text { Retired }\end{array}$ & $\begin{array}{c}24 \\
6 \\
4 \\
10 \\
6\end{array}$ & $\begin{array}{c}48.0 \\
12.0 \\
8.0 \\
20.0 \\
12.0\end{array}$ \\
\hline $\begin{array}{l}\text { 6. Duration of disease: } \\
-\quad \text { Less than one years } \\
-\quad \text { More than one year }\end{array}$ & $\begin{array}{c}7 \\
43\end{array}$ & $\begin{array}{l}14.0 \\
86.0\end{array}$ \\
\hline
\end{tabular}

Table (2) : Frequency distribution of studied patients according to health history $(n=50)$.

\begin{tabular}{|l|c|c|}
\hline \multicolumn{1}{|c|}{ Health history variables } & No. & \% \\
\hline Hypertension & 26 & 52.0 \\
\hline History of kidney disease & 6 & 12.0 \\
\hline Foot numbness & 22 & 44.0 \\
\hline Foot pain & 20 & 40.0 \\
\hline Footsore & 9 & 18.0 \\
\hline Tingling in feet & 22 & 44.0 \\
\hline
\end{tabular}

Table (3): Distribution of studied sample according to insulin type they use $(n=50)$.

\begin{tabular}{|l|c|c|}
\hline \multicolumn{1}{|c|}{ Type of insuline } & No. & \% \\
\hline Lantose & 5 & 10.0 \\
\hline Mixtrad anf hum line & 2 & 4.0 \\
\hline Lantose and hum line & 8 & 16.0 \\
\hline Mixtard & 11 & 22.0 \\
\hline Water insulin & 2 & 4.0 \\
\hline Water insulin and hum line & 4 & 8.0 \\
\hline Hum line & 18 & 36.0 \\
\hline
\end{tabular}


Table (4): Comparison between pre and post the implementation of health promotion life style booklet for studied patients regarding health responsibility pattern $(n=50)$.

\begin{tabular}{|c|c|c|c|c|c|c|c|c|c|c|c|c|c|c|c|c|c|}
\hline \multirow{3}{*}{ Health responsibility ( HR ) } & \multicolumn{8}{|c|}{ Pre } & \multicolumn{8}{|c|}{ Post } & \multirow{3}{*}{ p.v } \\
\hline & \multicolumn{2}{|c|}{ Never } & \multicolumn{2}{|c|}{ Sometimes } & \multicolumn{2}{|c|}{ Often } & \multicolumn{2}{|c|}{$\begin{array}{l}\text { Routin } \\
\text { ely }\end{array}$} & \multicolumn{2}{|c|}{$\begin{array}{c}\text { Neve } \\
\mathbf{r}\end{array}$} & \multicolumn{2}{|c|}{$\begin{array}{l}\text { Someti } \\
\text { mes }\end{array}$} & \multicolumn{2}{|c|}{ Often } & \multicolumn{2}{|c|}{$\begin{array}{c}\text { Routin } \\
\text { ely }\end{array}$} & \\
\hline & $\mathbf{N}$ & $\%$ & $\mathbf{N}$ & $\%$ & $\mathbf{N}$ & $\%$ & $\mathbf{N}$ & $\%$ & $\mathbf{N}$ & $\%$ & $\mathbf{N}$ & $\%$ & $\mathbf{N}$ & $\%$ & $\mathbf{N}$ & $\%$ & \\
\hline $\begin{array}{l}\text { 1. Report any unusual signs or symptoms } \\
\text { to a physician or other health } \\
\text { professional. }\end{array}$ & 5 & 10.0 & 18 & 36.0 & 15 & 30.0 & 12 & 24.0 & 0 & 0.0 & 3 & 6.0 & 17 & 34.0 & 30 & 60.0 & $\begin{array}{c}0.001 \\
* *\end{array}$ \\
\hline $\begin{array}{l}\text { 2. Read or watch TV programs about } \\
\text { improving health. }\end{array}$ & 16 & 32.0 & 18 & 36.0 & 10 & 20.0 & 6 & 12.0 & 1 & 2.0 & 6 & 12.0 & 22 & 44.0 & 21 & 42.0 & $\begin{array}{c}0.001 \\
* * \\
\end{array}$ \\
\hline $\begin{array}{l}\text { 3. Question health professionals in order } \\
\text { to understand their instructions. }\end{array}$ & 10 & 20.0 & 18 & 36.0 & 13 & 26.0 & 9 & 18.0 & 0 & 0 & 6 & 12.0 & 16 & 32.0 & 28 & 56.0 & $\begin{array}{c}0.001 \\
* *\end{array}$ \\
\hline $\begin{array}{l}\text { 4. Get a second opinion when I question } \\
\text { my health care provider's advice. }\end{array}$ & 20 & 40.0 & 17 & 34.0 & 8 & 16.0 & 5 & 10.0 & 0 & 0 & $\begin{array}{l}1 \\
8\end{array}$ & 36.0 & 23 & 46.0 & 9 & 18.0 & $\begin{array}{c}0.001 \\
* * *\end{array}$ \\
\hline $\begin{array}{l}\text { 5. Discuss my health concerns with health } \\
\text { professionals. }\end{array}$ & 6 & 12.0 & 18 & 36.0 & 14 & 28.0 & 12 & 22.0 & 0 & 0 & 3 & 6.0 & 9 & 18.0 & 38 & 76.0 & $\begin{array}{c}0.001 \\
* *\end{array}$ \\
\hline $\begin{array}{l}\text { 6. Inspect my body at least monthly for } \\
\text { physical changes/danger signs. }\end{array}$ & 22 & 44.0 & 22 & 44.0 & 5 & 10.0 & 1 & 2.0 & 0 & 0 & 3 & 6.0 & 32 & 64.0 & 15 & 30.0 & $\begin{array}{c}0.001 \\
* * \\
\end{array}$ \\
\hline $\begin{array}{l}\text { 7. Ask for information from health } \\
\text { professionals about how to take good } \\
\text { care of myself. }\end{array}$ & 8 & 16.0 & 21 & 42.0 & 12 & 24.0 & 9 & 18.0 & 0 & 0 & 2 & 4.0 & 15 & 30.0 & 33 & 66.0 & $\begin{array}{l}0.001 \\
* *\end{array}$ \\
\hline $\begin{array}{llll}\text { 8. Attend educational programs on } & \text { on } \\
\text { personal health care. } & & \\
\end{array}$ & 20 & 40.0 & 22 & 44.0 & 4 & 8.0 & 4 & 8.0 & 0 & 0 & 5 & 10.0 & 24 & 48.0 & 21 & 42.0 & $\begin{array}{c}0.001 \\
* *\end{array}$ \\
\hline $\begin{array}{l}\text { 9. Seek guidance or counseling when } \\
\text { necessary. }\end{array}$ & 1 & 2.0 & 17 & 34.0 & 20 & 40.0 & 12 & 24.0 & 0 & 0 & 0 & 0 & 12 & 24.0 & 38 & 76.0 & $\begin{array}{c}0.001 \\
* *\end{array}$ \\
\hline
\end{tabular}

Chi-Square Tests

$* *=$ highly significance,$* p \leq 0.01$

Table (5): Comparison between pre and post the implementation of health promotion life style booklet for studied patients regarding the physical activity pattern $(n=50)$.

\begin{tabular}{|c|c|c|c|c|c|c|c|c|c|c|c|c|c|c|c|c|c|}
\hline \multirow{3}{*}{ Physical activity ( PA) } & \multicolumn{8}{|c|}{ Pre } & \multicolumn{8}{|c|}{ Post } & \multirow{3}{*}{ p.v } \\
\hline & \multicolumn{2}{|c|}{ Never } & \multicolumn{2}{|c|}{$\begin{array}{c}\text { Someti } \\
\text { mes }\end{array}$} & \multicolumn{2}{|c|}{ Often } & \multicolumn{2}{|c|}{$\begin{array}{c}\text { Routine } \\
\text { ly }\end{array}$} & \multicolumn{2}{|c|}{ Never } & \multicolumn{2}{|c|}{$\begin{array}{c}\text { Someti } \\
\text { mes }\end{array}$} & \multicolumn{2}{|c|}{ Often } & \multicolumn{2}{|c|}{$\begin{array}{c}\text { Routine } \\
\text { ly }\end{array}$} & \\
\hline & $\mathbf{N}$ & $\%$ & $\mathbf{N}$ & $\%$ & $\mathbf{N}$ & $\%$ & $\mathbf{N}$ & $\%$ & $\mathbf{N}$ & $\%$ & $\mathbf{N}$ & $\%$ & $\mathbf{N}$ & $\%$ & $\mathbf{N}$ & $\%$ & \\
\hline $\begin{array}{l}\text { 2. Exercise vigorously for } 20 \text { or more minutes at least } \\
\text { three times a week such as ( brisk walking, bicycling, } \\
\text { aerobic dancing, using a stair climber). }\end{array}$ & 32 & 64.0 & 11 & 22.0 & 2 & 4.0 & 5 & 10.0 & 2 & 4.0 & 15 & $\begin{array}{c}30 . \\
0\end{array}$ & 19 & 38.0 & 14 & 28.0 & $\begin{array}{l}0.00 \\
1 * *\end{array}$ \\
\hline $\begin{array}{l}\text { 4. Take part in leisure-time (recreational) physical } \\
\text { activities (such as swimming, dancing, bicycling). }\end{array}$ & 43 & 86.0 & 6 & 12.0 & 0 & 0.0 & 1 & 2.0 & 10 & $\begin{array}{c}20 . \\
0\end{array}$ & 23 & $\begin{array}{cc}46 . \\
0\end{array}$ & 11 & 22.0 & 6 & 12.0 & \begin{tabular}{|l|}
0.00 \\
$1 * *$ \\
\end{tabular} \\
\hline 5. Do stretching exercises at least 3 times per week. & 31 & 62.0 & 11 & 22.0 & 5 & 10.0 & 3 & 6.0 & 2 & 4.0 & 6 & $\begin{array}{c}12 . \\
0\end{array}$ & 22 & 44.0 & 20 & 40.0 & \begin{tabular}{|l|}
0.00 \\
$1 * *$ \\
\end{tabular} \\
\hline $\begin{array}{l}\text { 6. Get exercise during usual daily activities (such as } \\
\text { walking during lunch, using stairs instead of elevators, } \\
\text { parking car away from destination and walking). }\end{array}$ & 20 & 40.0 & 20 & 40.0 & 5 & 10.0 & 5 & 10.0 & 3 & 6.0 & 3 & 6.0 & 22 & 44.0 & 22 & 44.0 & $\begin{array}{l}0.00 \\
1 * *\end{array}$ \\
\hline 7. Check my pulse rate when exercising. & 37 & 74.0 & 12 & 24.0 & 0 & 0.0 & 1 & 2.0 & 3 & 6.0 & 29 & $\begin{array}{c}58 . \\
0\end{array}$ & 11 & 22.0 & 7 & 14.0 & $\begin{array}{l}0.00 \\
1 * * \\
\end{array}$ \\
\hline 8. Reach my target heart rate when exercising. & 29 & 58.0 & 13 & 26.0 & 7 & 14.0 & 1 & 2.0 & 2 & 4.0 & 10 & $\begin{array}{c}20 . \\
0\end{array}$ & 25 & 50.0 & 13 & 26.0 & $\begin{array}{l}0.00 \\
1 * *\end{array}$ \\
\hline
\end{tabular}

Chi-Square Tests $\quad * *=$ highly significance,${ }^{*} p \leq 0.01$ 
Table (6): Comparison between pre and post the implementation of health promotion life style booklet for studied patients regarding interpersonal relations pattern $(n=50)$

\begin{tabular}{|c|c|c|c|c|c|c|c|c|c|c|c|c|c|c|c|c|c|}
\hline \multirow{3}{*}{$\begin{array}{l}\text { Interpersonal relations } \\
\text { (IR) }\end{array}$} & \multicolumn{8}{|c|}{ Pre } & \multicolumn{8}{|c|}{ Post } & \multirow{3}{*}{ P.v } \\
\hline & \multicolumn{2}{|c|}{ Never } & \multicolumn{2}{|c|}{ Sometimes } & \multicolumn{2}{|c|}{ Often } & \multicolumn{2}{|c|}{ Routinely } & \multicolumn{2}{|c|}{ Never } & \multicolumn{2}{|c|}{ Sometimes } & \multicolumn{2}{|c|}{ Often } & \multicolumn{2}{|c|}{ Routinely } & \\
\hline & $\mathrm{N}$ & $\%$ & $\mathrm{~N}$ & $\%$ & $\mathrm{~N}$ & $\%$ & $\mathrm{~N}$ & $\%$ & $\mathrm{~N}$ & $\%$ & $\mathrm{~N}$ & $\%$ & $\mathrm{~N}$ & $\%$ & $\mathrm{~N}$ & $\%$ & \\
\hline $\begin{array}{l}\text { 1. Discuss my problems and } \\
\text { concerns with people } \\
\text { close to me. }\end{array}$ & 5 & 10.0 & 7 & 14.0 & 22 & 44.0 & 16 & 32.0 & 0 & 0.0 & 0 & 0.0 & 7 & 14.0 & 43 & 86.0 & $0.001 * *$ \\
\hline $\begin{array}{l}\text { 2. Praise other people easily } \\
\text { for their achievements. }\end{array}$ & 4 & 8.0 & 13 & 26.0 & 23 & 46.0 & 10 & 20.0 & 0 & 0.0 & 2 & 4.0 & 18 & 36.0 & 30 & 60.0 & $0.001^{* *}$ \\
\hline $\begin{array}{l}\text { 3. Maintain meaningful and } \\
\text { fulfilling relationships } \\
\text { with others. }\end{array}$ & 1 & 2.0 & 11 & 22.0 & 23 & 46.0 & 15 & 30.0 & 0 & 0.0 & 0 & 0.0 & 13 & 26.0 & 37 & 74.0 & $0.001^{* *}$ \\
\hline $\begin{array}{l}\text { 4. Spend time with close } \\
\text { friends. }\end{array}$ & 0 & 0.0 & 25 & 50.0 & 14 & 28.0 & 11 & 22.0 & 0 & 0.0 & 2 & 4.0 & 28 & 56.0 & 20 & 40.0 & $0.001^{* *}$ \\
\hline $\begin{array}{l}\text { 5. Find it easy to show } \\
\text { concern, love and warmth } \\
\text { to others. }\end{array}$ & 0 & 0.0 & 19 & 38.0 & 19 & 38.0 & 12 & 24.0 & 0 & 0.0 & 4 & 8.0 & 14 & 28.0 & 32 & 64.0 & $0.001^{* *}$ \\
\hline $\begin{array}{l}\text { 6. Touch and am touched by } \\
\text { people I care about. }\end{array}$ & 11 & 22.0 & 13 & 26.0 & 16 & 32.0 & 10 & 20.0 & 3 & 6.0 & 5 & 10.0 & 23 & 46.0 & 19 & 38.0 & $0.001 * *$ \\
\hline $\begin{array}{l}\text { 7. Find ways to meet my } \\
\text { needs for intimacy. }\end{array}$ & 22 & 44.0 & 13 & 26.0 & 6 & 12.0 & 9 & 18.0 & 5 & 10.0 & 12 & 24.0 & 18 & 36.0 & 15 & 30.0 & $0.001 * *$ \\
\hline $\begin{array}{l}\text { 8. Get support from a } \\
\text { network of caring people. }\end{array}$ & 2 & 4.0 & 22 & 44.0 & 14 & 28.0 & 12 & 24.0 & 0 & 0.0 & 3 & 6.0 & 24 & 48.0 & 23 & 46.0 & $0.001 * *$ \\
\hline $\begin{array}{l}\text { 9. Settle conflicts with others } \\
\text { through discussion and } \\
\text { compromise. }\end{array}$ & 20 & 40.0 & 15 & 30.0 & 11 & 22.0 & 4 & 8.0 & 21 & 42.0 & 12 & 24.0 & 14 & 28.0 & 3 & 6.0 & $0.835 \mathrm{~ns}$ \\
\hline
\end{tabular}

Chi-Square Tests

$* *=$ highly significance,${ }^{*} p \leq 0.01 \quad N s=$ Non significant difference $p<0.05$

Table (7): Comparison between pre and post the implementation of health promotion life style booklet for studied patients regarding the nutritional pattern $(n=50)$.

\begin{tabular}{|c|c|c|c|c|c|c|c|c|c|c|c|c|c|c|c|c|c|}
\hline \multirow{3}{*}{ Nutrition (N) } & \multicolumn{8}{|c|}{ Pre } & \multicolumn{8}{|c|}{ Post } & \multirow{3}{*}{ p.v } \\
\hline & \multicolumn{2}{|c|}{ Never } & \multicolumn{2}{|c|}{ Sometimes } & \multicolumn{2}{|c|}{ Often } & \multicolumn{2}{|c|}{ Routinely } & \multicolumn{2}{|c|}{ Never } & \multicolumn{2}{|c|}{ Sometimes } & \multicolumn{2}{|c|}{ Often } & \multicolumn{2}{|c|}{ Routinely } & \\
\hline & $\mathbf{N}$ & $\%$ & $\mathbf{N}$ & $\%$ & $\mathbf{N}$ & $\%$ & $\mathbf{N}$ & $\%$ & $\mathbf{N}$ & $\%$ & $\mathbf{N}$ & $\%$ & $\mathbf{N}$ & $\%$ & $\mathbf{N}$ & $\%$ & \\
\hline $\begin{array}{l}\text { 1. Choose a diet low in fat, } \\
\text { saturated fat, and } \\
\text { cholesterol. }\end{array}$ & 10 & 20.0 & 18 & 36.0 & 18 & 36.0 & 4 & 8.0 & 3 & 6.0 & 3 & 6.0 & 28 & 56.0 & 16 & 32.0 .0 & $0.001^{* *}$ \\
\hline $\begin{array}{l}\text { 2. Limit use of sugars and } \\
\text { food containing sugar } \\
\text { (sweets). }\end{array}$ & 2 & 4.0 & 31 & 62.0 & 11 & 22.0 & 6 & 12.0 & 0 & 0.0 & 5 & 10.0 & 25 & 50.0 & 20 & 40.0 & $0.001^{* *}$ \\
\hline $\begin{array}{l}\text { 3. Eat } 6-11 \text { servings of } \\
\text { bread, cereal, rice and } \\
\text { pasta each day. }\end{array}$ & 2 & 4.0 & 20 & 40.0 & 26 & 32.0 & 2 & 4.0 & 1 & 2.0 & 19 & 38.0 & 19 & 38.0 & 11 & 22.0 & $0.053^{*}$ \\
\hline $\begin{array}{l}\text { 4. Eat } 2-4 \text { servings of fruit } \\
\text { each day. }\end{array}$ & 1 & 2.0 & 17 & 34.0 & 27 & 54.0 & 5 & 10.0 & 0 & 0.0 & 2 & 4.0 & 11 & 22.0 & 37 & 74.0 & $0.001^{* * *}$ \\
\hline $\begin{array}{l}\text { 5. Eat } 3-5 \text { servings of } \\
\text { vegetables each day. }\end{array}$ & 0 & 0.0 & 13 & 26.0 & 30 & 60.0 & 7 & 14.0 & 0 & 0.0 & 1 & 2.0 & 6 & 12.0 & 43 & 86.0 & $0.001^{* * *}$ \\
\hline $\begin{array}{l}\text { 6. Eat 2-3 servings of milk, } \\
\text { yogurt or cheese each } \\
\text { day. }\end{array}$ & 3 & 6.0 & 21 & 42.0 & 13 & 26.0 & 13 & 26.0 & 0 & 0.0 & 3 & 6.0 & 11 & 22.0 & 36 & 72.0 & $0.001^{* * *}$ \\
\hline $\begin{array}{l}\text { 7. Eat only } 2-3 \text { servings } \\
\text { from the meat, poultry, } \\
\text { fish, dried beans, eggs, } \\
\text { and nuts group each } \\
\text { day. }\end{array}$ & 1 & 2.0 & 16 & 32.0 & 26 & 52.0 & 7 & 14.0 & 0 & 0.0 & 3 & 6.0 & 17 & 34.0 & 30 & 60.0 & $0.001^{* * *}$ \\
\hline $\begin{array}{l}\text { 8. Read labels to identify } \\
\text { nutrients, fats, and } \\
\text { sodium content in } \\
\text { packaged food. }\end{array}$ & 32 & 64.0 & 8 & 16.0 & 5 & 10.0 & 5 & 10.0 & 8 & 16.0 & 18 & 36.0 & 12 & 24.0 & 12 & 24.0 & $0.001^{* * *}$ \\
\hline 9. Eat breakfast. & 1 & 2.0 & 2 & 4.0 & 7 & 14.0 & 40 & 80.0 & 0 & 0.0 & 0 & 0.0 & 3 & 6.0 & 47 & 94.0 & $0.160 \mathrm{~ns}$ \\
\hline
\end{tabular}

Table (8): Distributions of studied patients according to total scores of health promotion life style $(n=50)$

\begin{tabular}{|l|l|l|c|}
\hline \multicolumn{1}{|c|}{ Health promotion life style } & \multicolumn{1}{c|}{ Pre } & \multicolumn{1}{c|}{ Post } & P.V \\
\hline- Health responsibility & $20.66 \pm 5.57$ & $30.70 \pm 3.643$ & $0.001^{* *}$ \\
\hline- Physical activity & $12.82 \pm 4.27$ & $22.92 \pm 4.53$ & $0.001^{* *}$ \\
\hline- Interpersonal relations & $23.62 \pm 5.42$ & $29.48 \pm 3.47$ & $0.001^{* *}$ \\
\hline- Nutrition & $23.78 \pm 3.05$ & $30.48 \pm 2.55$ & $0.001^{* *}$ \\
\hline Total health promotion life style & $80.88 \pm 13.79$ & $113.58 \pm 11.80$ & $0.001^{* *}$ \\
\hline
\end{tabular}

Independent t-test $\quad * *=$ highly significance, ${ }^{*} p \leq 0.01$ 
Table (9): Relation between demographic data and life style patterns regarding the pre/ post-test of the studied sample $(n=50)$.

\begin{tabular}{|c|c|c|c|c|}
\hline \multirow{2}{*}{ Variables } & Pretest & Posttest & \multirow{2}{*}{$\begin{array}{c}\text { P1 } \\
\text { ( pretest) }\end{array}$} & \multirow{2}{*}{ P2 (posttest) } \\
\hline & Mean \pm SD & Mean \pm SD & & \\
\hline 1. Age: & & & \multirow{5}{*}{0.496} & \multirow{5}{*}{$0.015^{*}$} \\
\hline - $\quad 18$ less than $30 \mathrm{yrs}$ & $84.16 \pm 17.33$ & $120.66 \pm 7.50$ & & \\
\hline 30 less than $50 \mathrm{yrs}$ & $83.40 \pm 14.70$ & $118.46 \pm 9.01$ & & \\
\hline $50-65 y r s$ & $78.89 \pm 12.70$ & $109.58 \pm 12.37$ & & \\
\hline Total & $80.88 \pm 13.79$ & $113.58 \pm 11.80$ & & \\
\hline 2. Sex : & & & \multirow{3}{*}{0.318} & \multirow{3}{*}{$0.046^{*}$} \\
\hline - Male & $78.83 \pm 13.29$ & $110.12 \pm 13.50$ & & \\
\hline Female & $82.76 \pm 14.23$ & $116.76 \pm 9.12$ & & \\
\hline $\begin{array}{l}\text { 3. Level of education: } \\
-\quad \text { Illiterate }\end{array}$ & $78.00 \pm 14.27$ & $109.94 \pm 12.16$ & \multirow{7}{*}{0.302} & \multirow{7}{*}{$0.044^{*}$} \\
\hline - $\quad$ Read and write & $76.81 \pm 8.34$ & $108.09 \pm 14.06$ & & \\
\hline - $\quad$ Primary education & $77.33 \pm 6.35$ & $120.00 \pm 4.35$ & & \\
\hline Preparatory school & $80.00 \pm 0.00$ & $111.00 \pm 0.00$ & & \\
\hline Secondary school & $88.45 \pm 12.81$ & $120.18 \pm 5.17$ & & \\
\hline University & $86.40 \pm 22.76$ & $121.60 \pm 9.60$ & & \\
\hline Total & $80.88 \pm 13.79$ & $113.58 \pm 11.80$ & & \\
\hline $\begin{array}{l}\text { 4. Occupation: } \\
-\quad \text { Not-working }\end{array}$ & $80.00 \pm 10.23$ & $107.85 \pm 18.26$ & \multirow[t]{4}{*}{0.124} & \multirow[t]{4}{*}{0.252} \\
\hline - $\quad$ Farmer & $77.16 \pm 12.99$ & $109.83 \pm 7.93$ & & \\
\hline $\begin{array}{ll}- & \text { Student } \\
\end{array}$ & $92.00 \pm 12.30$ & $122.50 \pm 7.50$ & & \\
\hline $\begin{array}{ll}- & \text { Professional } \\
\end{array}$ & $82.00 \pm 16.14$ & $115.80 \pm 12.68$ & & \\
\hline - $\quad$ Retired & $68.66 \pm 6.47$ & $108.33 \pm 7.840$ & & \\
\hline Total & $80.88 \pm 13.79$ & $113.58 \pm 11.80$ & & \\
\hline
\end{tabular}

Tabel (1): Show that more than have of the studied sample (52\%) were female, the majority of the studied sample $(80 \%)$ were married, there age ranged from (50-65) years old, with mean age equal $49.220 \pm 14.226$. Regarding to the level of education, more than one third were not educated, and notworking $(48 \%)$. While the majority of the studied samples $(86 \%)$ were suffer from diabetes more than one year.

Table (2): Revealed that half of studied sample have hypertension $(52.0 \%)$ and less than half of them have foot numbness and tingling in feet $(44.0 \%)$.

Tabel (3): Mentioned that humline type used by more than one third $(36.0 \%)$ of the study group while less than one third $(22.0 \%)$ used mixtard insulin.

Table (4): This table illustrated that there were statistical significance difference between pre and post implementation of health promotion life style booklet for studied sample regarding to health responsibility pattern.

Table (5): This table illustrated that there were statistical significance difference between pre and post implementation of health promotion life style booklet for studied sample regarding to physical activity pattern.

Table (6): This table showed that there was statistical significance difference between pre and post implementation of health promotion life style booklet for studied sample regarding to interpersonal relations except Settle conflicts with others.

Table (7): This table showed that there was statistical significance difference between pre and post implementation of health promotion life style booklet in all items except regarding eating breakfast pattern.

Table (8): This table illustrated that according to total score of health promotion life style; there were statistical significance difference post implementation of health promotion life style booklet.

Table (9): This table reported that there were statistical significance difference in life style patterns post implementation of health promotion life style booklet in all element except in the occupation pattern.

\section{Discussion}

Type 1 diabetes formerly known as insulindependent in which the pancreas fails to produce the 
insulin which is essential for survival. This form develops most frequently in children and adolescents, but is being increasingly noted later in life. (WHO, 2016)

Lifestyle management is a fundamental aspect of diabetes care and includes diabetes self management education and support (DSMES), medical nutrition therapy (MNT), physical activity, smoking cessation counseling, and psychosocial care. Patients and care providers should focus together on how to optimize lifestyle from the time of the initial comprehensive medical evaluation, throughout all subsequent evaluations and follow-up, and during the assessment of complications and management of comorbid conditions in order to enhance diabetes (Piłaciński \& Zozulińska, 2014).

Regarding demographic characteristics, the present study revealed that female are more affected with type 1 diabetes than male; more than have of the studied sample were female, this result was in agreement with the study (MakkiAwouda et al, 2014) who found that the majority of participants were female.

In this study the age more than 50 years old, this is agreement with (MakkiAwouda et al., 2014) who found that major age group between 40-59. According to (CDC, 2017) who reported that the percentage of adults with diabetes increased with age, reaching more than one quarter among those aged 65 years or older years.

Regarding educational level among the studied sample the results revealed that more than one third were not educated. This result disagreed with (Torres et al., 2018) who reported that the majority of the studied sample was educated.

As regard the duration of disease the majority of the studied sample were suffer from diabetes more than one year. These may be related to the patients who were not diagnosed early with diabetes.

The present study revealed that regarding to the assessment of health history more than half of studied sample were have hypertension in my opinion these may be related to complication of diabetes. As diabetes decrease the blood vessels' ability to stretch and increasing the amount of fluid in the body and changing the way the body manages insulin, which may lead to increase blood pressure. These result were in agreement with (De Boer et al., 2017) who reported that hypertension is common and strong, risk factor for the macrovascular and microvascular complications of diabetes. According to (De Ferranti et al., 2014 ) who reported that hypertension is more common in patients with T1DM and is a powerful risk factor for cardio vascular diseases.

In the present study less than half of studied sample suffer from foot numbness and tingling in feet this result in agreement with (Tesfaye, 2011) who reported that diabetic peripheral neuropathy (DPN) is characterized by sensory loss and affects approximately half of people with diminished quality of life. According to (ADA, 2019) which recommended that patients with type 1 diabetes for more 5 years and all patients with type 2 diabetes should be assessed annually for diabetic peripheral neuropathy using the medical history and simple clinical tests. The most common early symptoms are induced by the involvement of small fibers and include pain and synesthesia (unpleasant sensations of burning and tingling sensation in the lower limp).

Regarding to insulin type more than one third of the study sample used hum line type and less than one third used mixtard insulin. This is reflect to hum line medication are avaliabel in the pharmacy and used in the hospital.

As regarding to health responsibility there was statistical significance difference between pre and post implementation of health promotion life style booklet for studied sample this result in agreement with (Hirjaba et al., 2015) who reported that patients responsibility have a positive impact on the quality of patients' lives and may promote health. The researcher opinion these difference refer to patients. As patients were more interested to get information and to perform healthy practices toward their disease and improving in their lifestyle.

Based on the results of the present study, there were statistical significance differences between pre and post implementation life style booklet for studied sample on improving physical activity pattern. This result is in the same line with (MakkiAwouda et al., 2014) Who found that there was statistical significance for exercise before and after the implementation of a health education program.

According to (Chimen et al., 2012) who mentioned that physical activity improves physical fitness and strength, reduces cardiovascular risk factors and improves well-being in type 1 diabetes, it also significantly reduces insulin requirements. According to ( ADA, 2018) recommendation: most adults with with type 1 diabetes should engage in $150 \mathrm{~min}$ or more of moderate-to-vigorous intensity physical activity per week, spread over at least 3 days/week, with no more than 2 consecutive days without activity.

Regarding to interpersonal relations, there were statistical significance difference between pre and post implementation of health promotion life style booklet for studied sample except settle conflicts with others. In the same line ( Trief et al., 2013) found that people with type 1 diabetes and their partners feel that the condition have an impacts on their relationship, posing both emotional and interpersonal 
challenges. So partner support is a vital source of support for those living with such a condition.

This study mentioned that regarding nutrition there were statistical significance differences between pre and post implementation of health promotion life style booklet in all items except eat breakfast this result in agreement with (MakkiAwouda et al., 2014). Who found that there was statistical significance relation between diet control and patients knowledge about importance of diet for diabetics before and after the implementation of the health education program.

The present study reported that according to total score of health promotion life style, there were statistical significance difference post implementation of health promotion life style booklet this result in agreement with (Amendezo et al., 2017) who mention that a structured lifestyle group education program for people with diabetes is an attractive option in a resource-limited setting, as it showed significant benefits in improveing glycemic control over a 12-month period.

\section{Conclusion}

Based on result, the study concluded that:

Most of patients with diabetes have unhealthy life style pattern. This leads to the appearance of some health problem and complications. There are statistical significance difference for studied samples as regard life style subscale in relation to health responsibility, physical activity, interpersonal relations and nutrition post the implementing of the life style promotion booklet.

\section{Recommendations}

\section{Recommendations for patients}

1. A continuing educations program should be planned and offered on regular basis for the patients and their families to explain the disease, its manifestation, complications and management.

2. Educational booklets and handouts should be available to teach patients and their families how to change their pattern of life.

3. Mass media should involve in providing the right and needed information about healthy patterns of lifestyle, in order to improve the level of knowledge among patients with diabetes.

4. Increase patients' awareness about the importance of healthy lifestyle and the extent of the effects on diabetes as a disease and its complications.

Recommendations for other researcher

1. Making aperiodic plan for each patient for his/her life style promotion education and follow up results.
2. More research should be carried out on a larger sample and health improvement programs should be disseminated in various centers for diabetic patients.

\section{References}

1. Amendezo, E., Timothy, D., Karamuka, V., Robinson, B., Kavabushi, P., Ntirenganya, C., \& Umulisa, H., (2017): Effects of a lifestyle education program on glycemic control among patients with diabetes at Kigali University Hospital, Rwanda: a randomized controlled trial. diabetes research and clinical practice, vol. (12) No. (6), Pp. 129-137.

2. American Diabetes Association. (2016): Classification and diagnosis of diabetes. Diabetes care, vol. (39) No. (1), Pp. 13-22.

3. American Diabetes Association. (2018): Classification and diagnosis of diabetes: standards of medical care in diabetes. Diabetes Care, vol. (41) No. (1), Pp. 13-27.

4. American Diabetes Association. (2019): Microvascular complications and foot care: Standards of Medical Care in Diabetes. Diabetes care, vol. (42) No. (1), Pp. 124-138.

5. Alpar, S., Senturan, L., Karabacak, U., \& Sabuncu, N., (2008): Change in the health promoting lifestyle behaviour of Turkish University nursing students from beginning to end of nurse training. Nurse Education in Practice, vol. (8) No. (6), Pp. 382-388.

6. Centers for Disease Control and Prevention. (2017): National diabetes statistics report, 2017. P. 14.

7. Chimen, M., Kennedy, A., Nirantharakumar, K., Pang, T., Andrews, R., \& Narendran, P., (2012): What are the health benefits of physical activity in type 1 diabetes mellitus. A literature review. Diabetologia, vol. (55) No. (3), Pp. 542551.

8. De Boer, I., Bangalore, S., Benetos, A., Davis, A., Michos, E., Muntner, P., \& Bakris, G., (2017): Diabetes and hypertension: a position statement by the American Diabetes Association. Diabetes Care, vol. (40) No. (9), Pp. 1273-1284.

9. De Ferranti, S., De Boer, I., Fonseca, V., Fox, C., Golden, S., Lavie, C., \& Zinman, B., (2014): Type 1 diabetes mellitus and cardiovascular disease: a scientific statement from the American Heart Association and American Diabetes Association. Circulation, vol. (130) No. (13), Pp. 1110-1130.

10. Garber, A., Abrahamson, M., Barzilay, J., Blonde, L., Bloomgarden, Z., Bush, M., \& 
Grunberger, G., (2013): American Association of Clinical Endocrinologists' comprehensive diabetes management algorithm consensus statement. Endocrine Practice, vol. (19) No. (2), Pp. 1-48.

11. Helsinki F., (1996): World medical association declaration of Helsinki, Recommendations guiding physicians in biomedical research involving human subjects, adopted by the 18th world medical assembly, P. 17.

12. Hirjaba, M., Häggman-Laitila, A., Pietilä, A., \& Kangasniemi, M., (2015): Patients have unwritten duties: experiences of patients with type 1 diabetes in health care. Health Expectations, vol. (18) No. (6), Pp. 3274-3285.

13. Kalyani, R., Cannon, C., Cherrington, A., Coustan, D., De Boer, I., Feldman, H., \& Neumiller, J., (2018): Professional Practice Committee: Standards of medical care in Diabetes. Diabetes Care, vol. (34) No. (15), Pp. 41-53.

14. MakkiAwouda, F., Elmukashfi, T., \& AlTom, S., (2014): Effects of health education of diabetic patient's knowledge at Diabetic Health Centers, Khartoum State, Sudan. Global journal of health science, vol. (6) No. (2), Pp. 221-230.

15. Noble, J., \& Valdes, A., (2011): Genetics of the HLA region in the prediction of type 1 diabetes. Current diabetes reports, vol. (11) No. (6), Pp. 533-540.

16. Ogurtsova, K., da Rocha Fernandes, J., Huang, Y., Linnenkamp, U., Guariguata, L., Cho, N., \& Makaroff, L., (2017): IDF Diabetes Atlas: Global estimates for the prevalence of diabetes for 2015 and 2040. Diabetes research and clinical practice, vol. (12) No. (8), Pp.40-50.

17. Ozougwu, J., Obimba, K., Belonwu, C., \& Unakalamba, C., (2013): The pathogenesis and pathophysiology of type 1 and type 2 diabetes mellitus. Journal of Physiology and Pathophysiology, vol. (4) No. (4), Pp. 46-57.

18. Pender, N., Murdaugh, C., \& Parsons, M., (2011): Health Promotion in Nursing Practice $6^{\text {th }}$ (ed). Boston, MA: Pearson. Pp. 189-196.

19. Pilaciński, S., \& Zozulińska-Ziólkiewicz, D., (2014): Influence of lifestyle on the course of type 1 diabetes mellitus. Archives of medical science: AMS, vol. (10) No. (1), Pp. 124.

20. Röder, P., Wu, B., Liu, Y., \& Han, W., (2016): Pancreatic regulation of glucose homeostasis. Experimental \& molecular medicine, vol. (48) No. (3), Pp. 219-225.

21. Tesfaye, S., (2011): Recent advances in the management of diabetic distal symmetrical polyneuropathy. Journal of diabetes investigation, vol. (2) No. (1), Pp. 33-42.

22. Torres, H., Pace, A., Chaves, F., VelasquezMelendez, G., \& Reis, I., (2018): Evaluation of the effects of a diabetes educational program: a randomized clinical trial. Revista de saude publica, Pp. 8-52.

23. Trief, P., Sandberg, J., Dimmock, J., Forken, P., \& Weinstock, R., (2013): Personal and relationship challenges of adults with type 1 diabetes: a qualitative focus group study. Diabetes Care, vol. (36) No. (9), Pp. 2483-2488.

24. Walker, S., Sechrist, K., \& Pender, N., (1987): The health-promoting lifestyle profile: development and psychometric characteristics. Nursing research, vol. (36) No. (2), Pp. 76-8.

25. Wei, C., Harada, K., Ueda, K., Fukumoto, K., Minamoto, K., \& Ueda, A., (2012): Assessment of health-promoting lifestyle profile in Japanese university students. Environmental health and preventive medicine, vol. (17) No. (3), P. 222.

26. World Health Organization. (2016): Global report on diabetes. P. 5. 